\title{
Inflation with a graceful exit and entrance driven by Hawking radiation
}

\author{
Sujoy Kumar Modak \\ IUCAA, Post Bag 4, Ganeshkhind, \\ Pune University Campus, Pune - $41100 \%$, India \\ Douglas Singleton $\bigsqcup^{\dagger}$ \\ Physics Department, CSU Fresno, \\ Fresno, CA 93740 USA \\ and \\ Institut für Mathematik, \\ Universität Potsdam Am Neuen Palais 10, \\ D-14469 Potsdam, Germany
}

(Dated: November 6, 2018)

\begin{abstract}
We present a model for cosmological inflation which has a natural "turn on" and a natural "turn off" mechanism. In our model inflation is driven by the Hawking-like radiation that occurs in Friedman-Robertson-Walker (FRW) space-time. This Hawking-like radiation results in an effective negative pressure "fluid" which leads to a rapid period of expansion in the very early Universe. As the Universe expands the FRW Hawking temperature decreases and the inflationary expansion turns off and makes a natural transition to the power law expansion of a radiation dominated universe. The "turn on" mechanism is more speculative, but is based on the common hypothesis that in a quantum theory of gravity at very high temperatures/high densities Hawking radiation will stop. Applying this speculation to the very early Universe implies that the Hawking-like radiation of the FRW space-time will be turned off and therefore the inflation driven by this radiation will turn off.
\end{abstract}

*Electronic address: sujoy@iucaa.ernet.in

${ }^{\dagger}$ Electronic address: $\overline{\text { dougs@csufresno.edu }}$ 


\section{INTRODUCTION}

Cosmological inflation [1] [2] [3] [4] was proposed to address the horizon problem, flatness problem and monopole problem in the context of Big Bang cosmology. By postulating that in the early Universe there was a brief period of rapid, exponential expansion one can explain, without fine-tuning, the observed facts that the Universe is the same in different regions which are causally disconnected (the horizon problem), the Universe appears to be spatially flat (the flatness problem) and that there appears to be a much lower density of Grand Unified monopoles than one would naively expect. However, the inflation hypothesis itself has several unanswered questions: (i) What is the detailed mechanism for inflation? (ii) What precedes the inflationary phase or how does inflation "turn on"? (iii) How does the Universe make a graceful exit from this early, inflationary phase to standard FriedmanRobertson-Walker (FRW) radiation dominated expansion i.e. how does inflation "turn off". In many of the original models [1] 3] [4] inflationary expansion was driven by a phase transition at the Grand Unified scale. The mechanism for inflation we propose here is based on particle creation from the gravitational field and it need not occur at the same time/energy scale compared to the canonical examples of inflationary mechanisms. Specifically, we focus on particle creation connected with the Hawking-like radiation that occurs in FRW spacetime. This is similar to black hole evaporation, but time reversed. For an astrophysical size black hole Hawking radiation is at first a very weak channel for mass/energy loss for the black hole. As the black hole decreases in mass due to loss from Hawking radiation it gets hotter and evaporates at a faster rate. Beyond some size Hawking radiation becomes very strong so that near the end stages of evaporation the black hole will radiate explosively. However, near the end stages of evaporation one can no longer trust the semi-classical calculation [5] leading to Hawking radiation. One common speculation is that near the end stages of evaporation where quantum gravity should become important, that Hawking radiation will "turn off". One concrete proposal along these lines is the suggestion that in the quantum gravity regime space-time becomes non-commutative which leads naturally to a turning off of Hawking radiation in the late stages of black hole evaporation [6]. Applying these ideas to FRW space-time leads to a time reversed version of black hole evaporation. During the very earliest stages of the Universe when the energy density is large, so that one is in the quantum gravity regime, the Hawking radiation from the FRW would be turned off until the 
Universe expanded to the point when quantum gravity started to give way to semi-classical gravity. At this point the Hawking radiation of FRW space-time would "turn on" and as we show below, would drive a period of exponential expansion. As the Universe expanded the Hawking temperature of the FRW universe would decrease until the Universe becomes dominated by ordinary radiation rather than Hawking radiation. At this point the Universe would make a gracefully transition from inflationary expansion to the power law expansion associated with a Universe dominated by ordinary radiation.

Already in the 1930s Schrödinger [7] put forward the idea that particle creation can influence cosmological evolution. More recently Parker [8] and others [9]-[16] have followed this early work of Schrödinger with studies of how particle creation can affect the structure of cosmological space-times. As pointed out in [14] there are two points about cosmological history which are well addressed by these particle creation models. First, one can explain very well the enormous entropy production in the early Universe via the irreversible energy flow from the gravitational field to the created particles. Second, since the matter creation is an irreversible process one avoids the initial singularity in cosmological space-times [14]. In this model the Universe begins from an instability of the vacuum instead of a singularity. The Universe then rapidly moves through an inflationary phase followed by a radiation dominated era and finally followed by a matter/dust dominated era.

\section{THERMODYNAMICS AND PARTICLE CREATION IN FRW SPACE-TIME}

Our particle creation/Hawking radiation model for inflation is closely tied to thermodynamics in a given space-time so we begin by collecting together some thermodynamic results. The first law of thermodynamics reads $d Q=d(\rho V)+p d V$, where $d Q$ is the heat flow into/out of the system during some interval of cosmic time from $t$ to $t+d t, \rho$ is the energy density, $V$ is the volume and $p$ is the thermodynamic pressure. Dividing this equation by $d t$, gives the following differential form for the first law of thermodynamics,

$$
\frac{d Q}{d t}=\frac{d}{d t}(\rho V)+p \frac{d V}{d t}
$$

For most cosmological models the assumption is made that the Universe is a closed, adiabatic system which means $d Q=0$. With this assumption the second law of thermodynamics, $d Q=T d S$, leads to a non-change in the entropy, i.e. $d S=0$, during the cosmic time interval 
$d t$. This line of reasoning contradicts the observed fact that the Universe has an enormous entropy. This contradiction can be addressed by having irreversible particle creation from the gravitational field i.e. Hawking radiation from an FRW space-time. This irreversible particle production leads to entropy production. The change in heat, $d Q$, is now completely due to the change of the number of particles coming from particle creation. Therefore there is a transfer of energy from the gravitational field to the created matter and the Universe is treated like an open, adiabatic thermodynamic system [14].

We review the relevant parts of the FRW space-time. The standard FRW metric is

$$
d s^{2}=-c^{2} d t^{2}+a^{2}(t)\left[\frac{d r^{2}}{1-k r^{2}}+r^{2}\left(d \theta^{2}+\sin ^{2} \theta d \phi^{2}\right)\right],
$$

where $a(t)$ is the scale factor and $k=0, \pm 1$ is the spatial curvature of the Universe $-k=0$ is flat, $k=-1$ is open and $k=+1$ is closed). The Einstein field equations $\left(G_{\mu \nu}=\frac{8 \pi G}{c^{4}} T_{\mu \nu}\right)$ for this metric have a time-time $(\mu=\nu=0)$ component and space-space $\mu=\nu=i$ component given respectively by

$$
3 \frac{\dot{a}^{2}}{a^{2}}+3 \frac{k c^{2}}{a^{2}}=\frac{8 \pi G \rho}{c^{2}}, \quad 2 \frac{\ddot{a}}{a}+\frac{\dot{a}^{2}}{a^{2}}+\frac{k c^{2}}{a^{2}}=-\frac{8 \pi G}{c^{2}} p .
$$

In the above equations $\rho$ is the energy density and $p$ is pressure of the matter source fluid/field. Combining these two equations gives the standard conservation relationship $d(\rho V)+p d V=0$, which clearly, describes the Universe as a closed, adiabatic system with $d Q=0$. As mentioned above this leads to $d S=0$ which then seems to contradict the very large observed entropy of the Universe. Allowing for matter creation alters things. First in the presence of matter creation the equations (3) are altered. The first equation on the left of (3) remains the same but the second equation is altered and one has an additional equation for the time rate of change of particle number density. These modified and additional equations are [17],

$$
\begin{aligned}
2 \frac{\ddot{a}}{a}+\frac{\dot{a}^{2}}{a^{2}}+\frac{k c^{2}}{a^{2}} & =-\frac{8 \pi G}{c^{2}}\left(p-p_{c}\right) \\
\frac{\dot{n}}{n}+3 \frac{\dot{a}}{a} & =\frac{\psi}{n} .
\end{aligned}
$$

The overdot implies a time derivative, $n$ is particle number density, $\psi$ is the matter creation rate and $p_{c}$ is the pressure due to matter creation. The matter creation rate and the matter creation pressure are connected by the following relationship [17],

$$
p_{c}=\frac{\rho+p}{3 n H} \psi
$$


If one assumes that $\rho$ and $p$ describe a normal fluid so that one has the energy condition $\rho+p>0$ (assuming that $\rho>0$ this condition is known as the weak energy condition [18]) and in addition that the matter creation rate is positive $\psi>0$ one can see that $p_{c}$ of (6) is positive and thus contributes a negative pressure to (4). Such negative pressures can drive accelerated expansion such as during the early inflationary phase of the Universe or during the current "dark energy" dominated era of the Universe. It would be economical if this negative pressure that occurs due to Hawking radiation in FRW space-time could drive both the inflationary era and the present accelerated phase of the Universe which is normally attributed to dark energy. We will show that while this particle creation pressure can drive inflation it can not drive the present accelerated expansion.

In the form (6) one could easily explain both inflation and the current accelerated expansion by simply choosing a matter creation rate $\psi$ to produce whatever acceleration (if $\psi>0$ ) or deceleration (if $\psi<0$ ) one wants. For example, if one wants exponential expansion, $a(t) \propto e^{H t}$ one should choose $\psi=3 n H$ [17]. However this choice has very little physical motivation beyond giving one the result one wanted in advance. The strength of our proposal is that the particle creation comes from a specific mechanism - Hawking radiation in FRW space-time - and as such leads to definite predictions which allow the model to be verified or ruled out. We will see that our mechanism does in fact lead to a particle production rate $\psi \approx 3 n H$.

We now move on to a discussion of Hawking radiation and associated temperature in FRW space-time. Since the FRW space-time is dynamical, the definition of the cosmological event horizon is subtle. However one can define the apparent horizon knowing the local properties of the space-time. In order to do this one can rewrite (2) in the following form [19]

$$
d s^{2}=h_{a b} d x^{a} d x^{b}+\tilde{r}^{2}\left(d \theta^{2}+\sin ^{2} \theta d \phi^{2}\right)
$$

where, $x^{a}=(t, r)$ and $h_{a b}=\operatorname{diag}\left(-c^{2}, a^{2} /\left(1-k r^{2}\right)\right)$ and $\tilde{r}=a(t) r$. The position of the apparent horizon is given by the root $\left(\tilde{r}_{A}\right)$ of the equation $\left(h^{a b} \partial_{a} \tilde{r} \partial_{b} \tilde{r}\right)_{\tilde{r}=\tilde{r}_{A}}=0$. Expanding this equation over $t, r$ sector and simplifying we get the position of the apparent horizon $\left(\tilde{r}_{A}\right)[20]$

$$
\left[h^{t t}\left(\partial_{t} \tilde{r}\right)^{2}+h^{r r}\left(\partial_{r} \tilde{r}\right)^{2}\right]_{\tilde{r}=\tilde{r}_{A}}=0 \Longrightarrow \tilde{r}_{A}=\frac{c}{\sqrt{H^{2}+\frac{k c^{2}}{a^{2}}}} .
$$


Using the above one can find the Hawking temperature of the apparent horizon [19]

$$
T=\frac{\hbar c \kappa}{2 \pi k_{B}}=\frac{\hbar c}{2 \pi k_{B}}\left(\frac{1}{\tilde{r}_{A}}\right)\left|1-\frac{\dot{\tilde{r}}_{A}}{2 H \tilde{r}_{A}}\right| .
$$

The first equality above is the standard relationship between the Hawking temperature and surface gravity $\kappa$ at the horizon of a given space-time. For FRW space-time the surface gravity is $\kappa=\frac{1}{\tilde{r}_{A}}\left|1-\frac{\dot{r}_{A}}{2 H \tilde{r}_{A}}\right|$. Thus in general the temperature, $T$, depends on both $\tilde{r}_{A}$ and its time derivative, $\dot{\tilde{r}}_{A}$. However, during an inflationary phase the Universe's scale factor takes the form $a(t) \propto \exp ($ constant $\times t)$ so that $H=\frac{\dot{a}}{a}=$ constant. If $H=$ constant satisfies $H^{2} \gg c^{2} / a^{2}$ (later we show this is the case for our model of inflation) we have from (8) $\tilde{r}_{A} \approx \frac{c}{H}=$ constant and $\dot{\tilde{r}}_{A} \approx 0$. Thus the temperature in (9) simplifies to[21]

$$
T=\frac{\hbar \sqrt{H^{2}+k c^{2} / a^{2}}}{2 \pi k_{B}} \approx \frac{\hbar H}{2 \pi k_{B}} .
$$

In the final approximation we are again assuming $H^{2} \gg c^{2} / a^{2}$ which as mentioned above we will justify later.

Before moving to a detailed calculation of how the particle creation pressure (6) affects the evolution of the early Universe in the case when this pressure comes from the particle creation from Hawking radiation, we give some numerical comparisons which show that this mechanism is of the correct order of magnitude to explain inflation. Considering $H$ to be inverse of the Planck time $\left(t_{p} \approx 10^{-43} s\right)$ gives from 110$) T \approx \frac{\hbar H}{2 \pi k_{B}} \approx 10^{32} \mathrm{~K}$. On the other hand at Planck energy $\left(E_{p}\right)$, gives a Planck temperature of $T_{p}=\frac{E_{p}}{k_{B}} \approx 10^{32} \mathrm{~K}$. Thus the Hawking temperature of FRW space-time at very early is around the Planck temperature. This large temperature associated with Hawking radiation of FRW spacetime in the early Universe is a good indication that our proposed mechanism has the proper order of magnitude to be a major factor in the early evolution of the Universe.

Our proposed Hawking radiation mechanism for inflation is the inverse of black hole evaporate. For astrophysical black holes the evaporation process begins very weakly - for a black hole having the mass of our Sun the temperature of the black body radiation emitted is $\approx 10^{-7} \mathrm{~K}$. However at the end stages of evaporation when the black holes has a small mass the evaporation will proceed explosively. At this point one is not justified in using the approximations that led to Hawking radiation as a thermal spectrum and it is said that one must have in hand a quantum theory of gravity to understand these end stages of black hole evaporation. For FRW space-time one is not justified in using Hawking radiation 
results at the very early stage of the Universe; one should have a theory of quantum gravity to understand this regime. As the Universe expands there will be a point at which the approximations leading to Hawking radiation from FRW space-time become valid. It is at this point that our Hawking radiation from FRW space-time mechanism for inflation "turns on" and inflation begins. As the Universe inflates further the Hawking temperature naturally decreases and our inflation mechanism will automatically "turn off".

One can see that our proposed process is the inverse of black hole evaporation since the direction of radiation flux of the Hawking radiation for the apparent horizon in a FRW space-time is the opposite from that of a Schwarzschild black hole event horizon. For black holes, the created particles escape outside the event horizon towards asymptotic infinity, while for the apparent horizon of FRW space-time the created particles come inward from the horizon. Due to the isotropy of FRW space-time, the radiation is isotropic from all directions. The net result is an effective power gain in the Universe, given by the StephanBoltzmann (S-B) radiation law. In summary the difference in the radiation direction from a Schwarzschild black hole and from the FRW space-time is as follows: for black holes the time rate of energy change $P$ is negative (i.e. they lose power during Hawking evaporation) while for the FRW space-time the time rate of energy change, $P$, is positive (i.e. the Universe gains energy). According to the Stephan-Boltzmann radiation law the time rate of energy gain due to Hawking radiation is

$$
P=+\frac{d Q}{d t}=\sigma A_{H} T^{4}
$$

where $\sigma=\frac{\pi^{2} k_{B}^{4}}{60 \hbar^{3} c^{2}}$ is the S-B constant and $A_{H}$ is the area of apparent horizon. Now one can substitute (11) into (1) but in that case the right hand side of (1) (which is the rate of change in energy flux through the apparent horizon) has to be evaluated at $\tilde{r}=\tilde{r}_{A}$, so that

$$
\left[\frac{d}{d t}(\rho V)+p \frac{d V}{d t}\right]_{\tilde{r}=\tilde{r}_{A}}=\sigma A_{H}\left(\frac{\hbar H}{2 \pi k_{B}}\right)^{4}
$$

where we have used (10). To calculate the left hand side we first consider the volume of a sphere of arbitrary radius by ignoring the curvature term i.e. we take $k=0$ and the volume is given by $V=\frac{4 \pi}{3} \tilde{r}^{3}=\frac{4 \pi}{3} r^{3} a^{3}(t)$. Note that here we take the radius at arbitrary $\tilde{r}$. Only after performing the $t$ derivative in $(12)$ we set $\tilde{r}=\tilde{r}_{A}$. On the other hand for the right hand side which represents the flow of energy across the apparent horizon we take $A_{H}=4 \pi \tilde{r}_{A}^{2}=4 \pi r_{A}^{2} a^{2}(t)$ where $\tilde{r}_{A}$ comes from (8). Using these expressions for the area and 
volume in 12 yields a modified continuity equation

$$
\dot{\rho}+3(\rho+p) \frac{\dot{a}}{a}=\frac{3 \sigma}{c}\left(\frac{\hbar}{2 \pi k_{B}}\right)^{4} H^{5},
$$

If one ignored the effect of the Hawking radiation/particle creation term on the right hand side of (13), by setting $T=0$ in (11), then $(13)$ becomes $\dot{\rho}+3(\rho+p) \frac{\dot{a}}{a}=0$ which is the usual continuity equation in the absence of particle creation.

Using $k=0$ and $H=\frac{\dot{a}}{a}$ we now rewrite (13) using the first equation in (3) as

$$
\frac{\dot{\rho}}{\rho}+3(1+\omega) \frac{\dot{a}}{a}=3 \omega_{c}(t) \frac{\dot{a}}{a},
$$

where we have taken the equation of state for ordinary matter as $p=\omega \rho$ and the time dependent equation of state due to particle creation is

$$
p_{c}(t)=\omega_{c}(t) \rho
$$

The equation of state parameter due to particle creation is

$$
\omega_{c}(t)=\alpha \rho(t), \text { where } \alpha=\frac{\hbar G^{2}}{45 c^{7}}=4.8 \times 10^{-116}\left(\mathrm{~J} / \mathrm{m}^{3}\right)^{-1}
$$

The constant $\alpha$ above is essentially the inverse of the Planck energy density $\rho_{\text {Planck }}=\frac{c^{7}}{\hbar G^{2}} \approx$ $10^{114}\left(\mathrm{~J} / \mathrm{m}^{3}\right)$. As we will show later it is this constant $\alpha$ sets the time and length scale for our inflation mechanism. This may also be different from the usual scale of inflation which is set by the Grand Unified scale. Moving the $\omega_{c}(t)$ term in 14 from the right hand side to the left hand side one can see that this particle creation term acts like a negative pressure. For the present Universe this term is negligible. The present value of the energy density of the Universe is $\rho_{0}=8.91 \times 10^{-10} \mathrm{~J} / \mathrm{m}^{3}$ so that $\omega_{c}\left(t_{0}\right)=\alpha \rho_{0} \approx 10^{-125}$ term on the right hand side of (14) is effectively zero. Thus this effective negative pressure can not explain the current accelerated expansion of the Universe - one still needs dark energy. However in the early Universe $\rho$ can be large enough so that the particle creation pressure on right hand side of (14) dominates, and as we will see this can drive inflation and also give a natural "turn off" for inflation.

At this point it should be mentioned that (14) does not violate Wald's first axiom [22, 23 on the energy-momentum tensor which is nothing but the usual conservation equation $\nabla_{\mu} T^{\mu \nu}=0$ [24, 25]. To see this, we note that in the absence of particle creation, the right hand side of (14) vanishes and the energy-momentum tensor has the form

$$
T^{\mu \nu}=\operatorname{diag}(\rho,-p,-p,-p)
$$


which satisfies the conservation equation. However in the presence of particle creation the above definition of $T^{\mu \nu}$ fails to simultaneously describe the conservation law and particle creation. In order to take both features into account one needs to consider a modification $T^{\mu \nu}$ which can deal with particle creation. Such a scenario is normally discussed in relationship to particle creation from black holes. Since, under the appropriate choice of vacuum state (i.e. the Unruh vacuum) black holes emit real particles in the form of thermal radiation, so that there is a power loss associated with Hawking radiation, it may appear that Wald's first axiom is violated. However, as demonstrated in [26], for such cases it is the regularized energy-momentum tensor $\left\langle T_{\nu}^{\mu}\right\rangle$ which satisfies the conservation equation $\nabla_{\mu}\left\langle T_{\nu}^{\mu}\right\rangle=0$. For the Unruh vacuum the regularized energy-momentum tensor is

$$
\left\langle T^{\mu \nu}\right\rangle=T_{(\text {gravitational })}^{\mu \nu}+T_{(\text {boundary })}^{\mu \nu}+T_{(\text {radiation })}^{\mu \nu}
$$

Thus it is clear that when one is dealing with particle creation it is the regularized (modified) energy-momentum tensor that satisfies Wald's axioms. This is exactly the picture in our case. Looking into the relation (14) one can see that the conservation equation in our case is given by $\nabla_{\mu} \tilde{T}^{\mu \nu}=0$, where the modified energy-momentum tensor has the form

$$
\tilde{T}^{\mu \nu}=\operatorname{diag}\left(\rho,-p^{\prime},-p^{\prime},-p^{\prime}\right)=T_{(\text {gravitational })}^{\mu \nu}+T_{(\text {radiation })}^{\mu \nu}(t) .
$$

In the above relation $p^{\prime}=p-p_{c}, T_{(\text {gravitational })}^{\mu \nu}$ is independent of time and given by (17) whereas the remaining part, $T_{(\text {radiation })}^{\mu \nu}(t)$, only contains the contribution from $p_{c}(t)-$ the particle creation pressure due to Hawking radiation.

In addition to the negative pressure (15) associated with particle creation due to Hawking radiation one can also calculate the effective particle creation rate, $\psi_{H}$, and compare with general result given in (6). Using the equation of state $p=\omega \rho$ one can re-write (6) as

$$
p_{c}=\frac{(1+\omega)}{3 n H} \psi \rho
$$

Equating (20) with (15) gives the time dependent matter creation rate associated with particle creation due to Hawking radiation in FRW space-time

$$
\psi_{H}(t)=\frac{3 n H \omega_{c}(t)}{(1+\omega)}
$$

Recall that in order to have exponential expansion $a(t) \propto e^{H t}$ one needs the creation rate from (6) to be $\psi \approx 3 n H$ [17]. Thus from (21), in order to have exponential expansion (i.e. 
inflation) one needs $\omega_{c}(t)$ to be approximately the same size as $1+\omega$ i.e. one needs $\omega_{c}(t) \approx \frac{4}{3}$ if one assumes the equation of state for ordinary radiation i.e. $\omega=\frac{1}{3}$. Since $\omega_{c}(t)=\alpha \rho(t)$ where $\alpha=4.8 \times 10^{-116}\left(\mathrm{~J} / \mathrm{m}^{3}\right)^{-1}$, this equality (i.e. $\left.\omega_{c}(t) \approx \frac{4}{3}\right)$ will occur when the density $\rho(t) \approx 10^{116}\left(\mathrm{~J} / \mathrm{m}^{3}\right)$ which is approximately the Planck density. This density corresponds to the density in the early Universe. Thus the rough calculations again point toward there being a large enough matter creation rate, $\psi_{H}(t)$, in the early Universe to drive inflationary expansion. However as the Universe expands and $\rho(t)$ drops the creation rate $\psi_{H}(t)$ will decrease and this Hawking radiation driven mechanism for inflation will turn off.

We now give a detailed calculation of inflation driven by Hawking radiation. Inserting $\omega_{c}(t)=\alpha \rho(t)$ into 14 one can integrate the resulting equation to find the energy density $\rho$ as a function of scale factor $a$

$$
\rho=\frac{D a^{-3(1+\omega)}}{1+\left(\frac{\alpha D}{1+\omega}\right) a^{-3(1+\omega)}} \rightarrow \frac{D a^{-4}}{1+\frac{3 \alpha D}{4} a^{-4}}=\frac{D}{a^{4}+\frac{3 \alpha D}{4}} .
$$

$D$ is a constant and in the last equality we have taken the equation of state of the ordinary matter to be that of radiation (i.e. $\omega=\frac{1}{3}$ ) since we want the early Hawking radiation inflation phase to be followed by a universe dominated by ordinary radiation. The dimensions of $D$ depend on the value of the equation of state parameter $\omega$. Note, in the classical limit $(\hbar \rightarrow 0), \alpha \rightarrow 0$, the FRW Hawking radiation effect turns off, and 22 gives $\rho \propto a^{-3(1+\omega)}$ which is the well known result for a universe dominated by ordinary matter with an equation of state $p=\omega \rho$.

There are two limits of this $\rho$ from 222): (i) $\alpha D \gg a^{4}$ so that $\rho \approx 4 /(3 \alpha)$ and the Hawking radiation effect dominates; (ii) $a^{4} \gg \alpha D$ so that $\rho \approx D / a^{4}$ which is the energy density of an ordinary radiation dominated universe. In case (i) the energy density is constant so that one has an effective cosmological constant which, as shown below, leads to exponential, inflationary expansion. In both cases (i) and (ii) the Universe is radiation dominated but for case (i) this means Hawking radiation of an FRW space-time and in case (ii) this means ordinary radiation. As one can see from the two limiting case behaviors of $\rho$ these two types of radiation result in very different evolution.

We now want to find the time-dependence of the scale factor $a(t)$. We begin by substituting $\rho$ (from (22) ) into the first equation in (3) to get a differential equation for $a$ as a function of $t$.(Recall we are assuming that $k$ in (3) is zero or negligible compared to the 
other terms). It is possible to integrate the resulting equation for $a$ to obtain

$$
\sqrt{\alpha D+\frac{4}{3} a^{4}}+\sqrt{\alpha D} \ln \left[\frac{a^{2}}{2 \sqrt{3}\left(\sqrt{\alpha D}+\sqrt{\alpha D+\frac{4}{3} a^{4}}\right)}\right]=\frac{8}{3} \sqrt{\frac{2 \pi G D}{c^{2}}} t-(K-1) \sqrt{\alpha D} .
$$

We have written the integration constant as $-(K-1) \sqrt{\alpha D}$ where $K$ is some positive number greater than 1 . This will make it easier to write out some of the later formulas. One important point to make about the scale factor, $a(t)$, in 23 is that it has an early exponential expansion phase (the second, logarithm term on the left hand side) which naturally transitions to a power law expansion (the first, power law term on the left hand side). We will discuss these two regimes in more detail in the following subsections. That these two phases come out naturally from the proposed inflation mechanism, without need for fine-tuning some inflaton potential, is a very attractive feature. In the next following three subsections we will analyze the the early time, exponential behavior of (23), the later time, power-law behavior of (23), and then we will discuss the possible values of $D$ and $K$.

\section{A. The very early Universe limit: $\alpha D \gg a^{4}$}

We first examine the limit of (23) in the very early Universe where $a(t)$ is of a size such that one has the limit $\alpha D \gg a^{4}$. In this limit $(23)$ becomes

$$
a(t)=2(3 \alpha D)^{\frac{1}{4}} \exp \left[\sqrt{\frac{32 \pi G}{9 c^{2} \alpha}} t-\frac{K}{2}\right] .
$$

Thus in this limit we find exponential expansion (inflation) with a Hubble constant given by

$$
H=\frac{\dot{a}}{a}=\sqrt{\frac{32 \pi G}{9 c^{2} \alpha}} \approx 10^{45} \frac{1}{s e c} .
$$

At this point we can return and justify some of our earlier assumption and approximations. First, after 12) we assumed that $H^{2} \gg k \frac{c^{2}}{a^{2}}$ is valid for $a(t)$ near the Planck size or larger (e.g. for $a \geq l_{p l}=10^{-35} \mathrm{~m}$ ). For $a(t)$ of the Planck scale one has $\frac{c^{2}}{a^{2}} \approx 10^{87}$ as compared to $H^{2} \approx 10^{90}$ from Eq. 25 . Second, we assumed that $\dot{\tilde{r}}_{A} \approx 0$. This is also justified since from (8) $\tilde{r}_{A} \approx \frac{1}{H}$ and during the inflationary phase $H$ is approximately constant with its value given by (25). Thus $\dot{\tilde{r}}_{A} \approx \frac{d}{d t}\left(\frac{1}{H}\right) \approx 0$. 
During inflation the standard lore is that the radius of the Universe should increase by a factor of $10^{26}$. Thus we need

$$
\frac{a\left(t_{\text {end }}\right)}{a\left(t_{\text {begin }}\right)}=10^{26}=\exp (H \Delta t),
$$

where $\Delta t=t_{\text {end }}-t_{\text {begin }}$, with $t_{\text {end }}, t_{\text {begin }}$ being the end and beginning time for this Hawking radiation driven inflation. From (25) we have $H=10^{45} \mathrm{sec}^{-1}$ so we find that (26) gives $\Delta t \approx 6 \times 10^{-44} \mathrm{sec}$. Note that if one took the ratio in 26 to be 10 orders larger (i.e. $\left.\frac{a\left(t_{\text {end }}\right)}{a\left(t_{\text {begin }}\right)}=10^{36}\right)$ this would yield $\Delta t \approx 8.3 \times 10^{-44}$ sec. In other words the time scale for the length of this inflation is set by $H$ in 25 and independent of $D$ and $K$ in (23). Because $H$ in 25) is so large one does not need a very long time, $\Delta t$, in order to inflate the Universe by many order of magnitude.

In contrast to the above mechanism of inflation, which is driven by near-Planck scale physics, the standard picture of inflation is that it is driven by physics at the Grand Unified scale i.e. by a Grand Unified phase transition. In this standard scenario inflation is thought to go from $t_{\text {begin }} \approx 10^{-36}$ sec. until $t_{\text {end }} \approx 10^{-33}$ sec. or $t_{\text {end }} \approx 10^{-32} \mathrm{sec}$. Thus for inflation driven by a phase transition at the Grand Unified scale one has $\Delta t \approx 10^{-33}-10^{-32}$ sec.
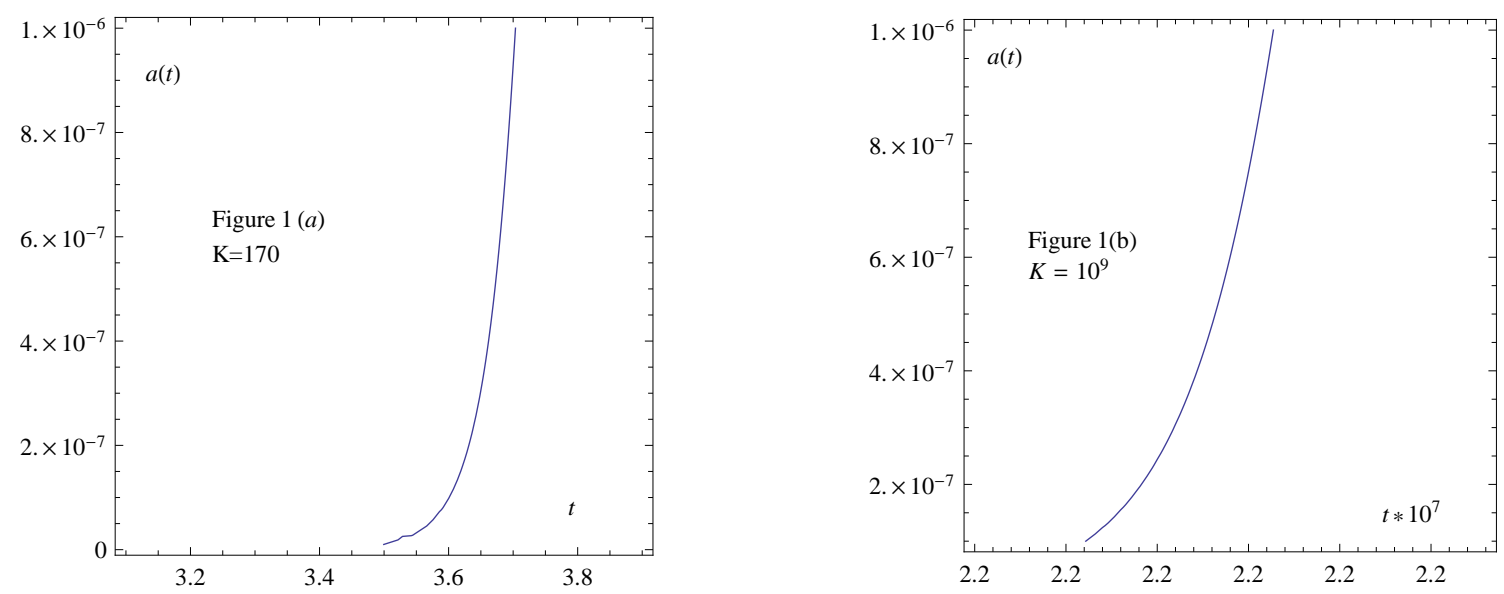

FIG. 1: Scale factor $a(t)$ is plotted with respect to $t$ (in units of Planck time $t_{P l}$ ) using equation (23). In (a) we fix $K=170$ and in (b) $K=10^{9}$. In both cases we take $D=10^{91} \mathrm{~J} / \mathrm{m}^{3}$. In this range of time $a(t)$ increases exponentially from Planck size to about $10^{-6}$ following the equation (24). Because of an extremely large value of the Hubble constant (25) the lifetime of this inflation is very small. This is the reason why in(b) apparently time is not changing along $x$ axis. In fact the change in $t$ takes place after the eight decimal places and thus does not appear in the plot. 
In (1) we show two plots of avs.t from (23) for the early, inflationary part of (23). In this figure we have set $D \approx 10^{91} \frac{\mathrm{J}}{\mathrm{m}^{3}}$ and two different values of $K$ are shown. This value of $D$ is justified in a subsequent section. From the two different values of $K$ we see that this parameter controls when inflation starts but it does not influence how long inflation last, which in this model is $\Delta t \approx 10^{-43}-10^{-44}$ sec.

\section{B. The not so early Universe limit: $a^{4} \gg \alpha D$}

The scale function $a(t)$ given in $(23)$ will leave the regime where the very early Universe approximation in (24) is valid, and then at some time will reach the point where $a(t) \approx$ $(\alpha D)^{\frac{1}{4}}$. After this intermediate stage $a(t)$ from $(23)$ will continue to increase until the regime is reached where $a^{4} \gg \alpha D$. In this limit $(23)$ gives

$$
\frac{2}{\sqrt{3}} a^{2}+(K-1) \sqrt{\alpha D}=\frac{8}{3} \sqrt{\frac{2 \pi G D}{c^{2}}} t
$$

Furthermore if the above condition is satisfied in a manner that $a^{2} \gg(K-1) \sqrt{\alpha D}$, one finally finds

$$
a(t) \approx\left(\frac{32 \pi G D}{3 c^{2}}\right)^{1 / 4} t^{1 / 2}
$$

This is the usual $t^{1 / 2}$ power law expansion for a radiation dominated universe. Thus after the inflationary stage given by (24) the solution given in (23) transitions into radiation dominated expansion given by 28$)$.

In (2) we show two plots of $a$ vs. $t$ from (23) which shows the beginning of the transition from exponential inflation to $t^{1 / 2}$ power law inflation. Again in this figure we have set $D \approx 10^{91} \frac{\mathrm{J}}{\mathrm{m}^{3}}$ and have the same values of $K$ as in (1). Again the two different values of $K$ control when inflation starts but they do not influence its duration.

\section{Determination of $D$ and $K$}

In this subsection we want to investigate possible values of the integration constants $D$ and $K . D$ can be set from the late time energy density of radiation. From [27] one finds that $\Omega_{\text {rad }}=\frac{\rho_{\text {rad }}}{\rho_{\text {crit }}} \approx 4.7 \times 10^{-5}$ which is the ratio of the radiation energy density to the critical

energy density. Using the value of the critical energy density $\left(\rho_{\text {crit }}=1.7 \times 10^{-9} \frac{\mathrm{J}}{\mathrm{m}^{3}}\right)$ we get $\rho_{\text {rad }}=8.0 \times 10^{-14} \frac{\mathrm{J}}{\mathrm{m}^{3}} \approx 10^{-13} \frac{\mathrm{J}}{\mathrm{m}^{3}}$ for the present radiation energy density. Equating this with 

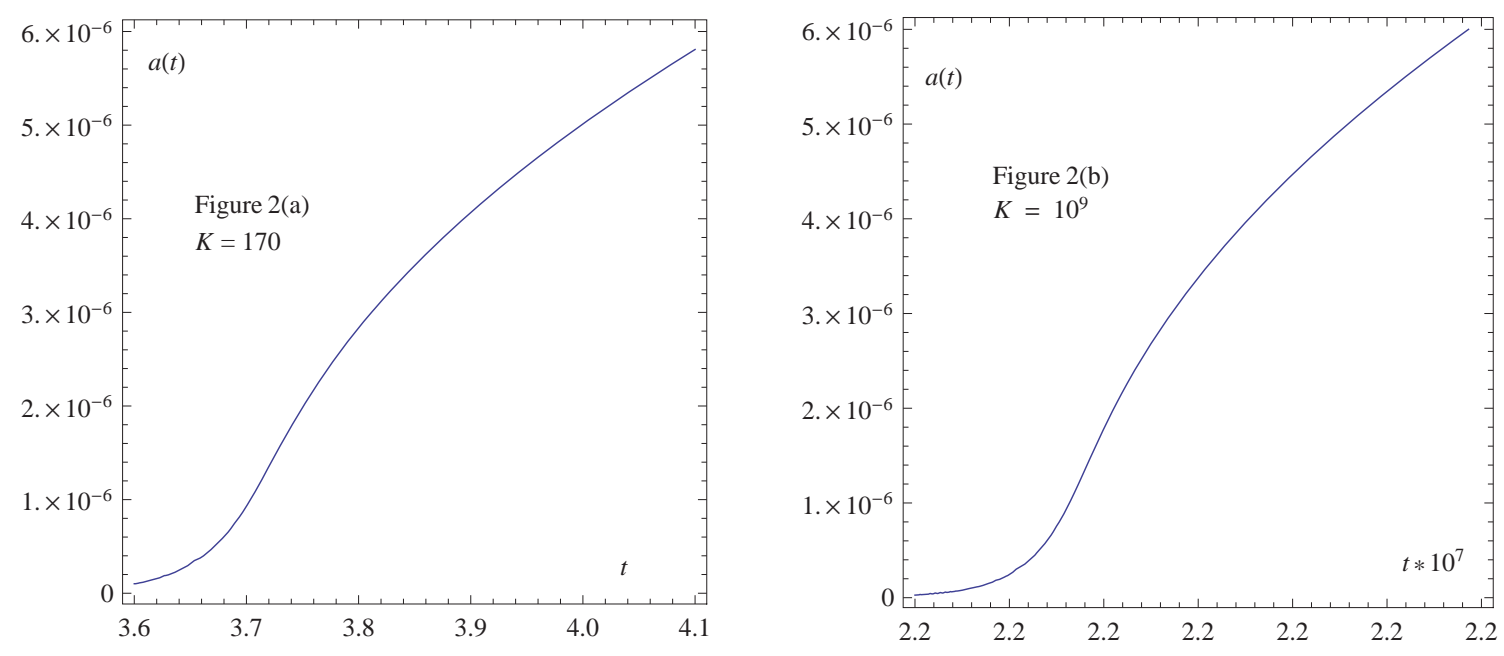

FIG. 2: Scale factor $a(t)$ is plotted with respect to $t$ (in units of Planck time $t_{P l}$ ) again by using (23). In (a) we consider $K=170$ and in (b) $K=10^{9}$. As before we take $D=10^{91} \mathrm{~J} / \mathrm{m}^{3}$ and time changes after eight decimal places in (b). Both figures show that in these intermediate values of $a(t)$ the inflationary behavior (24) naturally makes a transition to an ordinary radiation dominated era (28) for $a \approx 10^{-6}$. These figures nicely capture the end of inflation and the beginning of ordinary radiation domination.

$D / a^{4}$ and taking $a \approx 10^{26}$ as the present scale factor of the Universe yields $D \approx 10^{91} \frac{\mathrm{J}}{\mathrm{m}^{3}}$. Thus the amplitude of $(24)$ is $(\alpha D)^{\frac{1}{4}} \approx 10^{-6}$. Since for our inflationary phase scale factor $a(t)$ given by (24) we require $\alpha D \gg a^{4}$ (which because of the $4^{\text {th }}$ power can translate to $(\alpha D)^{\frac{1}{4}}>a$ ) we see that in this picture inflation stops at a scale of $>10^{-6}$ rather than $>0.1$. However given the uncertainty in when exactly inflation ends this is not a fatal problem. The scale of the Universe is still inflated by the same orders of magnitude - it just starts inflating a smaller scale and ends at a smaller scale.

Moving on to the constant $K$ one can see from Figs. 1 and 2 that this constant sets the time scale for when inflation starts. In plots $1(\mathrm{a})$ and $2(\mathrm{a})$ where $K$ is chosen to be $K=170$ we find that inflation starts at $t$ a few times larger than the Planck time $t_{P l}$. From the figures we see that for $K=170$ inflation starts at $3.4 t_{P l}$ to $3.8 t_{P l}$. On the other hand from plots $1(\mathrm{~b})$ and $2(\mathrm{~b})$ we see that for $K=10^{9}$ inflation starts at about $10^{7} t_{P l} \approx 10^{-36}$ sec. This start time corresponds to the standard picture where inflation is driven by a Grand Unified phase transition. Note that even though $K$ can shift the starting time of inflation, it can not control the duration which is fixed at $\Delta t \approx 10^{-43} \mathrm{sec}$. 


\section{GRACEFUL ENTRANCE TO INFLATION}

In the previous section we sketched a model for inflation driven by Hawking radiation of FRW space-time which has a natural "turn off" or graceful exit from inflation. We now offer speculation that this model of inflation driven by Hawking radiation may also have a natural "turn on" or entrance to inflation. As already noted the process for inflation suggested here is the reverse of black hole evaporation. During post-inflation (i.e. late stage) the Hawking radiation of FRW space-time will be a weak/minor effect just as Hawking radiation is weak/minor effect at the beginning (i.e. early stage) of black hole evaporation. During inflation (i.e. the very early stage) described in the section above the FRW Hawking radiation effect is dominate, just as during the end (i.e. very late stage) of black hole evaporation the Hawking radiation is dominate.

During the very late stages of evaporation of a black hole there are speculations that quantum gravity effects will turn off Hawking radiation. One particularly concrete example of this is in the non-commutative geometry scenario [6] where, as the Planck scale is approached, space-time become non-commutative

$$
\left[x^{\mu}, x^{\nu}\right]=i \theta^{\mu \nu}
$$

where $\theta^{\mu \nu}$ is an anti-symmetric rank 2 tensor which has the dimensions of distance squared. As a result of this non-commutativity black holes can not evaporate to arbitrarily small size, but due to the implied uncertainty relationship between spatial coordinates $-\Delta x^{i} \Delta x^{j} \geq$ $\frac{1}{2}\left|\theta^{i j}\right|$ for example $\Delta y \Delta z \geq \frac{1}{2}\left|\theta^{y z}\right|$ - a black hole can not shrink to zero size since then one would have $\Delta x^{i}=0$ in violation of this uncertainty relationship. Detailed analysis [6] shows that as a black hole evaporates in the non-commutative space-time characterized by (29) it reaches some maximum temperature after which the black hole temperature will decrease as the black hole continues to evaporate. At some point the Hawking temperature of the black hole goes to zero, the evaporation process stops and one is left with a non-radiating remnant [6]. Applying this picture to the FRW Hawking radiation model of inflation one would find that in the very early Universe, as during the late stages of black hole evaporation, the size of the Universe would be small and the FRW Hawking temperature would be zero. Thus at this early stage there would be no inflation since the FRW Hawking radiation would be "turned off". The Universe would expand "normally" according to a power law like (28). At some point the Universe would reach a size large enough not to be dominated 
by the uncertainty relationship coming from the noncommutative space-time relationship of 29. . At this point the FRW Hawking radiation would "turn on" and drive inflation until the Universe transitioned from the regime $\alpha D \gg a^{4}$ to the regime $a^{4} \gg \alpha D$. When the Universe entered this regime (i.e. $a^{4} \gg \alpha D$ ) it would undergo power law type of expansion given in 28) rather than the inflationary expansion of (24).

\section{SUMMARY}

In this paper we have proposed a mechanism for inflation based on the particle creation due to Hawing radiation in an FRW space-time. This mechanism differs from the model of inflation driven by some phase transition at the Grand Unified scale. This can be seen in the different time scales - inflation driven by a Grand Unified phase transition is thought to start at $t_{\text {begin }} \approx 10^{-36}$ sec. and last until $t_{\text {end }} \approx 10^{-33}-10^{-32}$ sec., thus having $\Delta t \approx 10^{-33}-10^{-32}$ sec. Because of the large value of $H$ in 25 (or alternatively the small value of $\alpha$ in $(16)$ ) the time scale of our proposed mechanism for inflation is $\Delta t \approx 10^{-44}-10^{-43}$ sec. which is different than the standard time for inflation. There are two constant, $D$ and $K$, which arise in the solution of the scale factor (23). The constant $D$ is determined by matching the theoretical late-time energy density $\left(\rho \approx D / a^{4}\right)$ with the observed value of the present day radiation energy density $\left(\rho_{\text {rad }} \approx 10^{-13} \frac{\mathrm{J}}{\mathrm{m}^{3}}\right)$ and the present day value of $a \approx 10^{26}$. In this way we obtain $D \approx 10^{91} \frac{\mathrm{J}}{\mathrm{m}^{3}}$. We also get a the amplitude of the inflationary period expression for $a(t)$ as given in $(24)$ namely $(\alpha D)^{1 / 4} \approx 10^{-6}$. This means that this model of inflation ends when $a \leq 10^{-7}$. This is six orders of magnitude smaller than the standard picture of inflation which ends at $a \approx 0.1$. However the scale factor in our model still inflates in size by a factor of $10^{26}$. In this picture inflation exits at a smaller scale factor than in the canonical picture. The other constant $K$ simply shifts when inflation starts, but does not control the duration. From Figs. 1 and 2 one can see for $K \approx \mathcal{O}(100)$ inflation starts near the Planck time while for $K \approx \mathcal{O}\left(10^{9}\right)$ inflations starts near $t \approx 10^{-36}$ sec - the standard starting time in inflation driven by a Grand Unified phase transition.

Because for some values of $K$ the starting time of inflation can be near the Planck time one should worry, for these values of $K$, about the validity of the calculation of the Hawking radiation. For one, near the Planck scale the constants $c, G$ and $\hbar$ could be different from the present day values. In particular since $\alpha$ in $(16)$ - and therefore $H$ in $(25)$ - depend on $c$ 
to the seventh power, having a different value of $c$ at these early, near-Planck times by even one order of magnitude would greatly change the scale of Hawking radiation driven inflation mechanism proposed here. If $c$ were one order of magnitude smaller in these very early times the energy scale of the Hawking radiation driven inflation would shift to be more in line with that of the Grand Unified phase transition mechanism for inflation. In this paper we simply stick to the simplest assumption - that $c, G$ and $\hbar$ - have the present, constant values even at these early, near-Planck times. We hope later to investigate the possibility that $c, G$ and/or $\hbar$ have different value at these early times.

In this picture of Hawking radiation driven inflation the time scale is set by $\alpha$ in (16). Setting aside this definite scale prediction for a moment - allowing for an arbitrary scale $\alpha$ - we note that one might regard (22), and the resulting scale factor $a(t)$ in (23), as a good phenomenological model for the time development of the size of the Universe which naturally includes exponential expansion with power law expansion in a single expression.

The inflation mechanism presented here is the time reversal of black hole evaporation. For a black hole in the early stages of evaporation via Hawking radiation, the radiation is a weak effect, barely changing the mass and space-time of the black hole; for an FRW universe in its late stages the Hawking radiation is a weak effect having effectively no effect on the expansion rate of the Universe. For a black hole in the late stages of evaporation via Hawking radiation, the radiation is a dominant effect, which plays a significant role in the change of the black hole's mass and the structure of the space-time; for an FRW universe in its early stages the Hawking radiation is a huge effect and leads to an enormous expansion rate (25) for the Universe. In the very late stages of black hole evaporation it is postulated that quantum gravity effects will shut off Hawking radiation; for an FRW space-time we postulate that in the very early stages quantum gravity effects will shut off Hawking radiation and the associated exponential expansion (24).

There have been other works that have studied the role of particle creation in the evolution of the Universe [7] - [17]. The present proposal is similar to the work of [14] which views particle creation as an irreversible process from energy transfer and entropy production from the gravitational field to the particles. The difference in the present work is that we have proposed a very specific particle creation mechanism namely the Hawking radiation associated with FRW space-time. The FRW Hawking radiation gives rise to an effective negative pressure evolution equation for the energy density, $\rho,(13)$ (14). The resulting $\rho$ 
given in (22) leads to a time dependent scale factor $a(t)$ given in $(23)$ which has two regimes one where $\alpha D \gg a^{4}$ with the resulting $a(t)$ being exponential/inflationary expansion as given in (24) and one where $a^{4} \gg \alpha D$ with the resulting $a(t)$ being power law expansion as given in (28). There is a natural transition from inflationary expansion to power law expansion so that this model for inflation has a graceful exit from inflationary behavior. Finally based on the inverse similarity between black hole evaporation and this FRW Hawking radiation model of the evolution of the scale factor $a(t)$, where the period of late time black hole evaporation corresponds to early period of the Universe (and visa versa), we have given some speculation as to how FRW Hawking radiation mechanism for inflation may "turn on" due to non-commutative space-time effects. Thus the FRW Hawking radiation picture for the evolution of $a(t)$ provides not only a graceful exit to inflation as well as a possible graceful entrance.

One final comment - this inflation mechanism has a feedback mechanism which forces the scale factor, $a(t)$, to be uniform. For example, if one assumed that the scale factor also had a dependence on $r$ (i.e. $a(r, t))$ the Hawking radiation inflation mechanism would tend to erase this $r$ dependence. If, $a(r, t)$ were smaller for some $r$ this would imply a higher Hawking temperature and more rapid expansion. This would push those regions of $r$ with smaller scale factor, $a$, to expand more rapidly until they were the same as the scale factor in other regions. If, $a(r, t)$ were larger for some $r$ this would imply a lower Hawking temperature and less rapid expansion. This would push those regions of $r$ with larger scale factor, $a$, to expand less rapidly until they were the same as the scale factor in other regions.

Acknowledgment: DS is supported by a DAAD grant.

[1] A. H. Guth, Phys. Rev. D23, 347 (1981)

[2] A.A. Starobinsky, Phys. Lett. B91, 99 (1980)

[3] A. Linde, Phys. Letts. B 108, 389 (1982)

[4] A. Albrecht and P. Steinhardt, Phys. Rev. Letts. 48, 1220 (1982)

[5] S.W. Hawking, Comm. Math. Phys. 43, 199 (1975).

[6] P. Nicolini, Int. J. Mod. Phys. A24, 1229 (2009).

[7] E. Schrödinger, Physica (Amsterdam) 6, 899 (1939). 
[8] L. Parker, Phys. Rev. Lett. 21, 562 (1968).

[9] R. Brout, F. Englert and E. Gunzig, Ann. Phys. 115, 78 (1978).

[10] R. Brout, F. Englert and E. Gunzig, Gen. Relativ. Gravit. 10, 1 (1979).

[11] R. Brout, F. Englert and E. Gunzig, Nucl. Phys. B170, 228 (1980).

[12] R. Brout, F. Englert and P. Spindel, Phys. Rev. Lett. 43, 417 (1979).

[13] P. Glansdorff and I. Prigogine, "Thermodynamic Theory of Structure, Stability and Fluctuations," Wiley Interscience, New York, (1971).

[14] I. Prigogine, J. Geheniau, E. Gunzig, P. Nardone, Gen. Relativ. Gravit. 21, 767 (1989).

[15] C. P. Singh, A. Beesham, Astrophys. Space Sci. 336, 469 (2011).

[16] C. Gao, Entropy 14, 1296 (2012)

[17] J. A. S. Lima, A. S. M. Germano and L. R. W. Abramo, Phys. Rev. D53, 4287 (1996).

[18] M. Visser "Lorentizian Wormholes", (Springer-Verlag, New York, 1996)

[19] R. Cai, S. P. Kim, JHEP 0502, 050 (2005).

[20] D. Bak and S. Rey, Class. Quant. Grav. 17, L83 (2000)

[21] T. Zhu, J.R. Ren, and D. Singleton, Int. Mod. Phys. D19, 159 (2010).

[22] R.M. Wald, Commun. Math. Phys. 54, 1 (1977).

[23] R.M. Wald, Phys. Rev. D 17, 1477 (1978).

[24] J. V. Narlikar, "An introduction to Cosmology," Cambridge University Press, (2002).

[25] T. Padmanabhan, "Gravitation-Foundations and Frontiers," Cambridge University Press, (2010).

[26] T. Christodoulakis, G. A. Diamandis, B. C. Georgalas and E. C. Vagenas, Phys. Rev. D 64, 124022 (2001) hep-th/0107049].

[27] J. Beringer et al. (Particle Data Group), Phys. Rev. D86, 010001 (2012) 